\title{
大脑皮层内活动依赖的神经环路结构可塑性研究进展
}

\author{
饶小平 1,3 , 许智祥 1,3 , 徐富强 1,2 , \\ (1. 中国科学院武汉物理与数学研究所 波谱与原子分子物理国家重点实验室, 武汉 430071; \\ 2. 武汉光电国家实验室, 武汉 430074; 3. 中国科学院大学, 北京 100049)
}

\begin{abstract}
摘要: 哺乳动物大脑皮层内的神经环路在神经发育、学习记忆、神经和精神疾病过程中表现出令人惊异的结 构和功能可塑性。随着新的成像技术及分子生物学方法的应用，在细胞和突触水平上观察活体皮层内神经环路的 动态结构变化成为可能，因此近十年来有关活动依赖的神经环路结构可塑性方面的研究进展迅速。该文综述了该 方面的部分实验结果, 重点阐述个体生长发育、丰富环境、感觉剥夺、病理状态以及学习和记忆等过程和条件下 树突的结构可塑性特点, 尤其是树突棘的形态和数量变化特征; 并简单介绍轴突的结构可塑性, 以及结构可塑性 相关的分子和细胞机制，最后提出未来该领域内亟待解决的问题。
\end{abstract}

关键词: 神经环路; 可塑性; 成像技术; 生长发育; 丰富环境; 感觉剥夺; 病理状态; 学习和记忆; 树突的结构 可塑性; 树突棘; 轴突的结构可塑性; 分子和细胞机制

中图分类号: Q4, Q189; 文献标志码: A 文章编号: 0254-5853-(2012)05-0527-10

\section{Progress in activity-dependent structural plasticity of neural circuits in cortex}

\author{
RAO Xiao-Ping ${ }^{1,3}$, XU Zhi-Xiang ${ }^{1,3}$, XU Fu-Qiang ${ }^{1,2, *}$ \\ (1. State Key Laboratory of Magnetic Resonance and Atomic and Molecular Physics, Wuhan Institute of Physics and Mathematics, the Chinese \\ Academy of Sciences, Wuhan 430071, China; 2. Wuhan National Laboratory for Optoelectronics, Wuhan 430074, China; \\ 3. University of the Chinese Academy of Sciences, Beijing 100049, China)
}

\begin{abstract}
Neural circuits of mammalian cerebral cortex have exhibited amazing abilities of structural and functional plasticity in development, learning and memory, neurological and psychiatric diseases. With the new imaging techniques and the application of molecular biology methods, observation neural circuits' structural dynamics within the cortex in vivo at the cellular and synaptic level was possible, so there were many great progresses in the field of the activity-dependent structural plasticity over the past decade. This paper reviewed some of the aspects of the experimental results, focused on the characteristics of dendritic structural plasticity in individual growth and development, rich environment, sensory deprivation, and pathological conditions, as well as learning and memory, especially the dynamics of dendritic spines on morphology and quantity; after that, we introduced axonal structural plasticity, the molecular and cellular mechanisms of structural plasticity, and proposed some future problems to be solved at last.
\end{abstract}

Key words: Neural circuits; Plasticity; Imaging techniques; Growth and development; Rich environment; Sensory deprivation; Pathological conditions; Learning and memory; Dendritic structural plasticity; Dendritic spine; Axonal structural plasticity; Molecular and cellular mechanism

神经环路由神经元的轴突、树突以及连接两者 的突触构成。轴突将神经元信息输出到不同的区域, 有的甚至可以跨越几乎整个大脑; 树突将来自于可 达几百微米的不同来源的输入信息进行整合; 突触
则是神经环路中进行信息传递、交换以及计算的位 置(Holtmaat \& Svoboda, 2009)。神经环路可塑性涉 及突触可塑性、树突可塑性、轴突可塑性以及神经 细胞自身可塑性等(Mozzachiodi \& Byrne, 2010), 其

\footnotetext{
收稿日期: 2012-07-04; 接受日期: 2012-08-30

基金项目: 国家自然科学基金杰出青年基金(08Q1011001); 中国科学院百人计划(08B1021001); 武汉光电国家实验室创新基金(Z08004); 中国科 学院武汉物理与数学研究所重点资助项目 $(08 \mathrm{~K} 1011001)$

*通信作者(Corresponding author), Tel: 027-87197091, E-mail: fuqiang.xu@wipm.ac.cn
} 
中突触的可塑性是整个神经环路可塑性的核心。典 型的突触是由带有突触囊泡的突触前活性区 (presynaptic active zone)、突触间隙(cleft)以及突触 后致密区(postsynaptic density, PSD)构成(Holtmaat \& Svoboda, 2009)。突触的可塑性包括突触的功能可 塑性(即已经存在的突触连接强度的增加或减弱)以 及突触的结构可塑性 (即突触的形成或消 亡)(Holtmaat \& Svoboda, 2009)。突触的重要性使其 在过去的几十年中一直备受关注, 目前我们对突触 的功能可塑性相关现象及其分子机制已经有较深 的理解(Ho et al, 2011)。而突触结构可塑性在最近十 年内, 由于活体双光子显微延时成像(two-photon microscopy and time-lapse imaging)、双光子苂光寿 命显微成像(two-photo fluorescence lifetime imaging microscopy, 2 pFLIM) 、菼光共振能量转移 (fluorescence resonance energy transfer, FRET)成像 以及三维重建连续切片透射式电子显微镜图像 (serial section transmission electron microscopy)技术 的运用, 结合大脑神经元中稀疏携带 GFP 或 YFP 等荧光蛋白的转基因动物的应用, 开启了揭示活体 内神经环路结构可塑性与功能可塑性之间联系以 及结构可塑性的细胞和分子机制的研究(Lendvai et al, 2000; Patterson \& Yasuda, 2011)。本文仅综述该方 面的最新进展。

\section{1 树突的可塑性}

神经元的树突是接受从其它神经元传入信息 的入口。树突的轴干上分布着许多微小的突起, 这 些突起在形态、大小、密度以及动态特征方面各不 相同，在不同的发育阶段以及不同的神经类型中也 存在着差异; 虽然对树突突起的分类还存在争议, 但是一般突起可分为树突棘 (spine) 和丝状伪足 (filopodia): 前者指较短的突起, 具有球状头部, 虽 然大小不一(包括细的棘、短而粗的棘和蘑菇状的棘; 如图 1)(Amaral \& Pozzo-Miller, 2009), 但基本上都 能形成具有功能的突触结构; 后者指树突上细长的 突起, 无球状头部, 一般不形成功能性的突触, 但 有些可以转变为棘 $(\mathrm{Fu} \&$ Zuo, 2011; Holtmaat \& Svoboda, 2009)。由于在成年动物大脑中, 树突分枝 保持的比较稳定，丝状伪足总数<总的突起的 $10 \%$, 且其形成或消亡的时间尺度以分钟或小时计，因此 树突的结构可塑性主要是指树突棘的可塑性(Zuo et al, 2005a)。树突棘的可塑性一般包括形态和数量的
改变。形态改变指树突棘头部几何形态的变大或变 小; 数量改变则指树突棘的形成或消亡, 表现为棘 的更新与密度的变化。

树突棘形态或尺寸的变化以及数量的改变具 有怎样的生理意义? 在正常情况下，成熟大脑中 10\%的棘具有不成熟的形态：短而粗、多突触、具 丝状伪足或分叉; 突触活动的增加或减少可以引起 细的棘发生改变, 因此被认为是“学习棘”; 而蘑菇 状的棘具有稳定性，因此被称为“记忆棘”; 突触活 动增强能导致细的棘转变成蘑菇状的棘(Bourne \& Harris, 2007)。树突棘的体积与功能紧密相关: 蘑菇 状的棘拥有较宽的 PSD, 含有较多功能性 AMPA 型 谷氨酸受体以及可以产生较强的兴奋性突触后电 位等(Kasai et al, 2010)。长时程增强(LTP)与长时程 抑制(LTD) 分别与树突棘的长期增长和萎缩相关 (Park et al, 2006; Zhou et al, 2004)。因此棘的尺寸与 突触强度相关, 棘的形态变化反应突触强度的变化 (Matsuzaki et al, 2004)。树突棘数量的增加或减少伴 随着突触的更新，表明神经环路的连接发生了变化 (Trachtenberg et al, 2002); 相对突触连接强度的变 化, 新突触的形成可能更大程度地增加了大脑的记 忆储存能力(Chklovskii et al, 2004); 此外，除了对 原有树突棘的修饰作用，新的树突棘形成也与某些 形式的 LTP 有关(Engert \& Bonhoeffer, 1999; Toni et al, 1999)。因此，树突棘形态的变化与突触强度的改 变相关, 而树突棘数量的变化则反应了神经连接的 改变(Yuste \& Bonhoeffer, 2004)。

\section{1 个体成长发育过程中的树突可塑性}

Cajal 以及后来一系列脑组织固定观察实验发 现，哺乳动物，包括人，出生后早期大脑皮层均有 突触的快速形成，紧接着出现大量的树突棘/突触 丢失 $(\sim 50 \%)$, 直至青春期结束，而成年期树突棘保 持相对稳定，但也存在着与年龄相关的突触丢失 (Bhatt et al, 2009)。双光子活体延时成像发现，年轻 小鼠(一月龄)的视皮层、体感皮层、初级运动皮层 和前额叶皮层在间隔 2 周的时间内有 $13 \%$ 20\%的 棘消失以及 $5 \%$ \% 的棘形成，表明动物在发育期 存在着广泛的树突棘丢失(Trachtenberg et al, 2002; Zuo et al, 2005a)。也有发现表明树突棘的净丢失在 2 周龄时即已开始(Holtmaat et al, 2005)。随着动物 逐渐发育成熟(四月龄以前), 由于消失速度远高于 形成速度，所以，欶的数量净损失 $25 \%$ (Zuo et al, $2005 \mathrm{a})$ 。在成年阶段(四月龄以后)，小鼠视皮层 V 

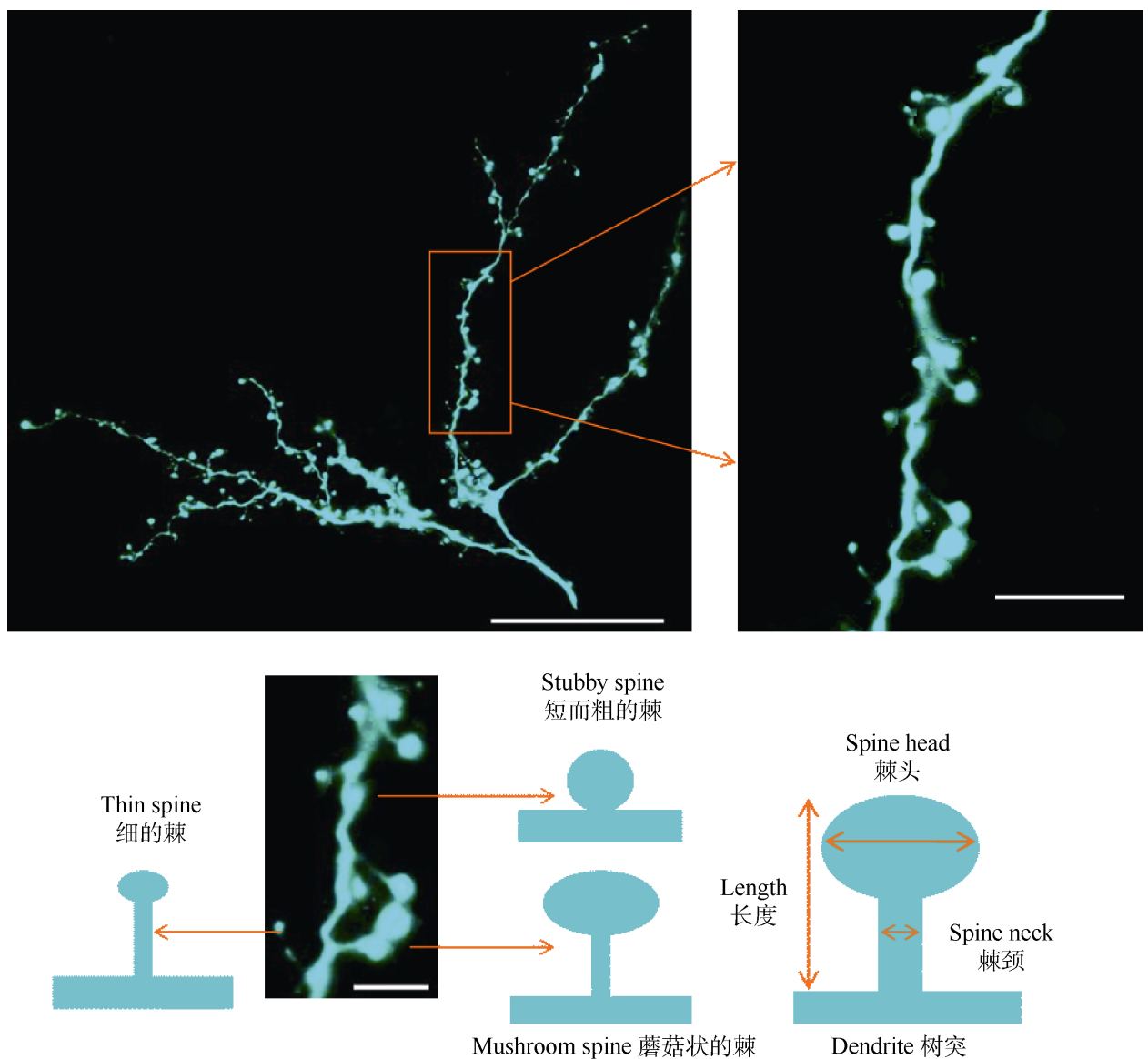

图 1 小鼠嗅球中颗粒细胞上不同类型的树突棘[修改自 Amaral \& Pozzo-Miller (2009)]

Fig. 1 Different types of dendritic spines of granule cells in the olfactory bulb of mice [Modified from Amaral \& Pozzo-Miller (2009)] 上图) C57 小鼠嗅球内颗粒细胞顶树突在不同放大倍数下的显微图像，可见树突分支的复杂性以及在第二级和第三级分支上存在着大量的树突棘。下 图左侧）图片叠加后可以发现树突轴干上分布着最常见的三种形态的树突棘：细的棘、短而粗的棘和蘑菇状的棘; 下图右侧）树突棘的分类标准常 用头部直径、颈部直径以及整体长度等几何尺寸指标来定性和定量描述。上图左侧、右侧以及下图左侧的标尺分别为: $50 \mu \mathrm{m} 、 10 \mu \mathrm{m}$ 和 $5 \mu \mathrm{m}$ 。

Top panels: the apical dendrites of granule cell in the olfactory bulb of C57 mice are shown at different magnifications to illustrate the complexity of their dendritic arbors, as well as the abundance of dendritic spines on the secondary and tertiary branches. Bottom left panel: after image stack, we can find that dentritic segment was studded with the most common spine morphologies, such as thin, stubby and mushroom; Bottom right panel: the classification criteria of dendritic spine are commonly used in head diameter, neck diameter, overall length and other geometric dimensions to describe them qualitatively and quantitatively. Top left panel: Scale bar $=50 \mu \mathrm{m}$, Top right panel: Scale bar $=10 \mu \mathrm{m}$, Bottom left panel: Scale bar $=5 \mu \mathrm{m}$.

层雉体神经元的顶树突棘相当稳定，在 1 个月内有 $\sim 4 \%$ 的更新(Grutzendler et al, 2002); 而在小鼠体感 皮层、初级运动皮层和前额叶皮层中有 $3 \% \sim 5 \%$ 的 棘形成或消失, $70 \%$ 的树突棘能够稳定存在 18 个月, 且棘形成和消亡的速度相似(Zuo et al, 2005a)。但也 有发现表明成年阶段的体感皮层树突棘在 8 天时间 内更新 40\%(Trachtenberg et al, 2002)。这可能是由 于所使用动物的年龄、品系以及是否进行开颖手术 等不同所造成(Bhatt et al, 2009)。丝状伪足占树突轴 上突起的比例随年龄逐渐下降: 在小鼠柱状皮层中, 其比例从二周龄的 $>50 \%$ 下降到一月龄的 $<10 \%$; 到 成年阶段, 其比例进一步下降至 2\% 3\% (Zuo et al, 2005a)。

出生后的发育过程中，柱状皮层内树突棘的消 亡速度下降, 形成速度保持不变, 因而树突棘的总 量开始减少，之后保持稳定，并与年龄表现出一种 单指数方程的函数关系 $\left(70.0 \%+30.0 \% \times 0.5^{\mathrm{t} / 15.63 \mathrm{~d}}\right.$, $\left.\chi^{2}=8.4\right)$ : 一至二月龄的动物中, $32 \%$ 的树突棘消亡, 其平均半衰期为 11.3 天，而剩余 $68 \%$ 的树突棘可以 保持稳定不变; 相反, 如果所有树突棘消亡的速度 一致，成年(四月龄)动物树突棘的平均半衰期则为 13.2 个月(Grutzendler et al, 2002)。树突棘从短半衰 期到长半衰期的转变, 可能与关键期的结束有关; 树突棘的稳定性可能经历着从关键期内较强的可 
塑性转变为成年阶段较弱的可塑性的过程 (Grutzendler et al, 2002)。

受双光子穿透深度所限, 活体观察得到的数据 有限。不同物种不同脑区不同层以及不同细胞类型 的树突棘/突触数量变化都可能不同。如成年小鼠的 嗅球和海马雉体神经元的树突分支或树突棘均可 保持相对稳定(Mizrahi et al, 2004; Mizrahi \& Katz, 2003)。总而言之, 在动物出生后的早期发育过程中, 大脑皮层的突触密度迅速增加，随后大量丢失，直 至青春期结束; 而在成年阶段, 突触数量则保持相 对稳定直至生命终止。因此, 不论任何发育阶段, 都有小部分树突棘长期维持稳定，这可能是哺乳动 物皮层发育的普遍规律; 因而也为基本皮层功能和 终生记忆储存提供了结构基础(Yang et al, 2009)。虽 然绝大部分树突棘在成年阶段能够长期存在, 但树 突棘的长度与头部直径等形态特征在发育阶段和 成年阶段均存在着可塑性变化(Grutzendler et al, 2002; Holtmaat et al, 2005; Zuo et al, 2005a), 由于棘 的尺寸与突触强度相关, 棘的形态变化反应突触强 度的变化, 所以这种形态变化可能为短时间的信息 储存提供了结构基础, 也可能参与了快速可塑性, 如伤害和经验引起的皮层神经环路的改变 (Matsuzaki et al, 2004)。这种突触效能的改变可能会 最终调控树突棘在较长时间内的形成和消亡, 以及 神经环路的长期改变(Grutzendler et al, 2002)。

\section{2 丰富环境下的树突可塑性}

丰富环境是研究感觉经验对神经可塑性作用 的一个定性非常清楚的模型。丰富环境的研究一般 有两种动物饲养方式: (1)空间限制、环境单调、笼 子小以及无社会性交往等; (2)大笼子, 有玩具、巢 穴、障碍物、群养以及给予充分的解决问题的机会 和复杂的社交环境等(Alvarez \& Sabatini, 2007)。因 此, 丰富环境涵盖感觉刺激, 学习, 运动, 社交等 多种成分。

丰富环境可以提高认知表现和学习, 提高海马 的 LTP, 影响基因表达、细胞增殖和存活率, 以及突 触形成和神经元形态(Alvarez \& Sabatini, 2007)。在 神经元形态方面, 丰富环境能增加皮层和海马某些 区域的雉体神经元树突的分叉、树突棘的密度以及 突触的数量, PSD 的数量和平均长度, 以及位于树 突棘头部的多聚核糖体的数量。丰富环境对活动依 赖可塑性的影响在所有年龄阶段均有表现, 甚至出 生前的丰富环境也能提升大鼠的某些特异性行为
(Alvarez \& Sabatini, 2007)。

在小鼠的柱状皮层中, 丰富环境可以让先前存 在的树突棘变得不稳定：据估计，丰富环境下有 $\sim 73 \%$ 先前存在的树突棘能从一月龄维持到二月龄, 低于标准环境 $5 \%$; 四月龄成年小鼠在丰富环境中 有 $83 \%$ 的旧棘能存在 5 个月，低于标准环境 3\%; 丰富环境下 $90 \%$ 旧棘的平均寿命为 71 个月，低于 标准环境 19 个月; 丰富环境下 37\%在一月龄时存 在的棘能持续到动物生命的终点, 低于标准环境 11\%(Yang et al, 2009)。另外, 丰富环境可以引起新 的树突棘形成， $<0.8 \%$ 的新树突棘在丰富环境中可 以存在 80 个月, 而在标准的饲养环境中可以存在 73 个月, 即丰富环境可以使新形成的树突棘更为稳 定(Yang et al, 2009)。丰富环境对树突棘可塑性的影 响与学习训练的效果相同(Yang et al, 2009)。

\section{3 感觉剥夺后的树突可塑性}

活动经验对神经连接模式以及行为的影响将 贯穿动物一生是神经系统的一个基本特征, 经验/ 神经活动对突触形成, 尤其是神经环路的构建具有 关键作用(Bhatt et al, 2009)。在青春期(一月龄), 剪 掉一侧的全部胡须(感觉剥夺)能降低柱状皮层内顶 树突棘的消亡速度，而对树突棘的形成没有影响， 从而可以阻止树突棘的净丢失, 表现为树突棘数量 或密度的增加; 而恢复动物的胡须感觉后树突棘加 速消亡; 且在丝状伪足中未发现类似现象 (Zuo et al, $2005 b$ )。以上结果说明经验/神经活动依赖的神经连 接的修饰过程对哺乳动物神经系统的发育成熟极 其重要(Bhatt et al，2009)，且该效应随动物的成熟 而减弱。例如，在树突棘逐渐趋于稳定的成年期(四 月龄以上), 2 周时间的感觉剥夺对柱状皮层内棘的 形成或丢失无显著影响, 但是长期感觉剥夺 (2 个月) 则会引起树突棘消失速度的轻微下降 $(23 \%)$ (Zuo et $a l, 2005 b)$ 。而采用剃掉单个胡须保持周边完整的棋 盘剥夺方式, 有的结果相同(Zuo et al, 2005b), 有的 结果却表明感觉剥夺无论是在青春期还是成年期 都只影响棘的动态性而不影响棘密度; 剃掉胡须能 增加新形成的树突棘的稳定性，而降低长期存在的 树突棘的稳定性。因此, 感觉剥夺使旧的树突棘失 去稳定而使新的树突棘变得稳定，总的效果为增加 树突棘的动态性，导致突触连接变化更快(Holtmaat et al, 2006; Trachtenberg et al, 2002)。

黑暗饲养、单眼剥夺 $(\mathrm{MD})$ 和双眼剥夺 $(\mathrm{BD})$ 为研 究视觉皮层可塑性普遍采用的三种模型。黑暗饲养 
会引起动物树突棘密度的下降; 关键期内 4 天的 $\mathrm{MD}$ 可以引起小鼠视皮层第 $\amalg$ 层顶部和底部树突 棘的快速消失(Mataga et al, 2004)。虽然棘的形态变 化和更新随着年龄的增长而下降, 但是感觉剥夺在 发育过程中对棘的动态性的影响在不同的皮层内 可能有所差别。在体感皮层的快速突触形成时期 (P11-P13), 剃须会导致 II/III 层神经元树突棘的形 态变化程度下降(Lendvai et al, 2000), BD 却能在视 皮层关键期的顶峰时期(P28)提高 $\mathrm{V}$ 层树突棘的形 态变化程度(Majewska \& Sur, 2003), 而且不同深度 皮层的树突棘形态具有不同的变化特点(Oray et al, 2004), 树突棘这种层次特异性的变化特征可能是 由处于不同位置的特异性分子构成所决定(Mataga et al, 2004; Oray et al, 2004)。因此, 对于中枢的某个 神经元, 感觉刺激只能不同程度地影响特定区域范 围内相关的神经连接(Fox \& Wong, 2005)。

总之, 感觉经验/神经活动对树突棘/突触净丢 失的作用几乎贯穿动物一生, 尤其是青少年时期 (Zuo et al, 2005b); 成年期感觉剥夺引起的可塑性 与关键期的可塑性在某些方面具有相似性, 虽然前 者需要更长的剥夺时间, 说明关键期在某些方面并 非骤然结束, 而是以渐进的形式趋向关闭状态 (Alvarez \& Sabatini, 2007)。

\section{4 病理条件下的树突可塑性}

树突棘的形态和数量与神经功能联系紧密, 改 变树突棘的形态结构以及干扰树突棘的形成或消 亡等都可能对神经环路产生影响, 甚至导致一系列 认知功能障碍(Bhatt et al, 2009)。许多神经和精神疾 病都与树突棘形态或密度的大量改变有关。例如, 脆性 X 综合症(FXS), 是一种最普遍的遗传性智障, 虽然具有较高的树突棘浓度, 但多数为不成熟的、 又长又细的棘, 不能发育为蘑菇状的棘, 且更新速 度较快, 状态不稳定, 不受感觉经验的操控(Pan et al，2010), 同时表现出由代谢型谷氨酸受体调控的 LTD 的增强 (Bear et al，2004); 而三染色体 21(trisomy 21), 也叫蒙古症(Mongolism)或唐氏症 (Down syndrome), 是一种染色体异常的病变, 通常 会造成心智障碍, 大脑新皮层和海马区的树突棘密 度一般比较低(Kaufmann \& Moser, 2000)。此外, 树 突棘形态和数量的异常也常常出现在其它的大脑 神经紊乱或疾病中, 如成瘾、恐惧、抑郁、朊病毒 病或蛋白粒子病、癫㾁、缺血症和中风等(Bhatt et al, 2009)。
树突棘的结构变化也可能是许多与年龄有关 的神经退行性疾病的发病机理, 如阿尔兹海默症 (Alzheimer's disease, $\mathrm{AD}$ )。通过活体观察 $\mathrm{AD}$ 小鼠 和 YFP 小鼠的杂交后代发现，淀粉样蛋白沉积能诱 发附近神经元结构的持续改变, 不论是穿越还是靠 近淀粉样蛋白沉积斑块的神经元均表现某种程度 上的树突棘丢失和树突轴直径下降 (Tsai et al, 2004)。针对年龄增长引起的认知功能下降的治疗方 案可以引起细的树突棘数量特异性增加(Hao et al, 2006), 表明在衰老的大脑中需要细的树突棘以重 建或恢复突触可塑性与学习潜力(Bourne \& Harris, 2007)。各种大脑神经疾病伴随着树突棘形态或密度 的巨大变化, 充分说明了树突棘的动态变化在正常 环境中是保持动态平衡的, 但是这种状态很容易受 到不同病理条件下的多种因素影响而失衡(Bhatt et al, 2009)。

\section{5 学习和记忆过程中的树突可塑性}

通过双光子延时成像活体观察运动技能学习 小鼠运动皮层的雉体神经元顶树突的结构变化发 现, 无论是在青春期还是成年期, 学习均可引起新 树突棘的快速形成，同时也导致先前存在的旧树突 棘变得不稳定并快速消亡(如图 2b); 虽然棘的形成 速度高于消亡，但是持续时间较短，因而树突棘总 量保持不变(如图 2c)(Xu et al, 2009; Yang et al, 2009; Ziv \& Ahissar, 2009)。新棘形成快, 消亡也快, 但是 重复训练可以使新形成的树突棘变得更加稳定, 训 练结束后也能持续较长时间(Xu et al, 2009)。小鼠进 行运动技能训练后三个月, 即使 $30 \%$ $40 \%$ 新形成 的棘发生消亡(或突触丢失), 依然能维持先前学习 的运动技能; 新形成的棘存在的越多, 记得越牢, 行为表现越好; 同时, 行为表现也与旧棘的消亡相 关，消亡越多，行为表现也越好(Yang et al，2009); 说明大多数稳定的神经环路依然能保留先前学习 的技巧(Yang et al，2009); 训练后的复习阶段虽然 没有新棘的形成, 但是学习新技能时仍然能引起新 棘的大量形成(如图 2d)(Xu et al, 2009; Yang et al, 2009; Ziv \& Ahissar, 2009), 说明不同的运动技能引 起不同的棘形成和消亡，即不同的记忆编码在不同 的突触群中(Xu et al, 2009; Ziv \& Ahissar, 2009); 此 外, 长时间重复训练可以引起 $1 / 3$ 新形成的棘成簇 状或相邻分布, 相对分散分布的新形成的棘, 簇状 分布的新棘具有较高的稳定性, 倾向于出现在与旧 棘相邻近的空白位置上，而分散分布的新棘在树突 
(a) Before learning 学习前

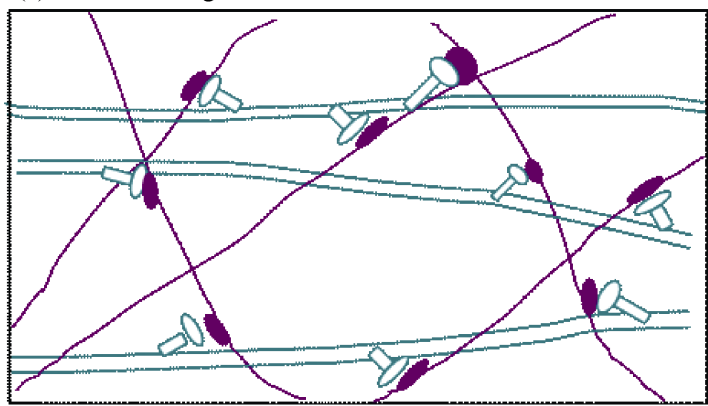

(c) Learning consolidation 学习巩固

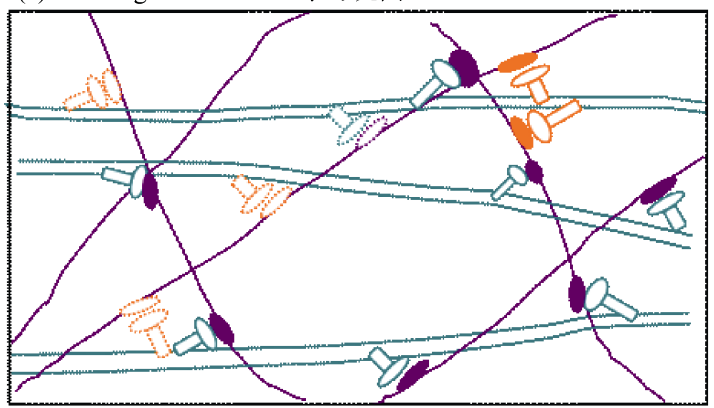

(b) Learning task A 学习任务 A

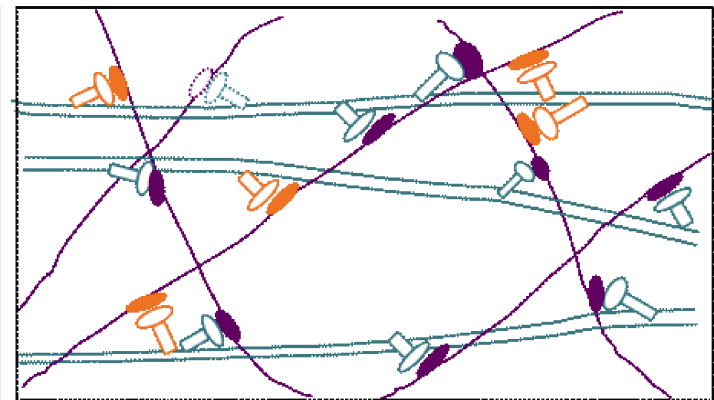

(d) Learning task B 学习任务 B

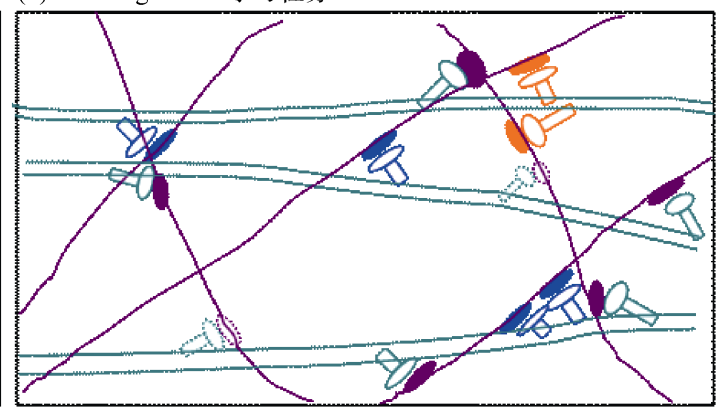

图 2 伴随着运动学习，小鼠运动皮层内树突棘的动态变化及神经回路的重组[修改自 Fu \& Zuo（2011)]

Fig. 2 The dynamics of dendritic spines and circuits remodelling associated with motor task learning in motor cortex of mice

[Modified from Fu \& Zuo (2011)]

a) 轴突中途结(紫色)与树突的突起(树突棘, 绿色实线)形成突触; b)学习一个运动技能(任务 A)伴随着新的树突棘的形成(桔红色实线)以及旧的树突棘 的消失(绿色虚线); ) 学习任务 $\mathrm{A}$ 后的记忆巩固过程中, 旧的以及新形成的树突棘(桔红色虚线)均有消失; d) 学习新的运动技能(任务 B)能引起新的树突 棘形成(蓝色实线)以及旧的树突棘消失，而对在之前运动技能学习(任务 A)过程中新形成的树突棘没有影响。

a. Synapse consists of axonal boutton (purple) and the neighboring dentritic spine (green); b. Learning a motor skill (task A) is accompanied by the formation of new dendritic spines (orange-red, solid line) and the disappearance of the old spines (green, dotted line); c. After the learning task A, old (green, dotted line) and new spines (orange-red, dotted line) disappeared in the process of memory consolidation; d. Learning task B could induce new spines formation (blue, solid line) and old spines disappearance, but don’t affect the new spines formed in motor skill learning (task A) previously.

上均匀分布, 会避免与旧棘靠近(如图 $2 \mathrm{~b}, \mathrm{c})$; 这说 明记忆在单个神经元上的储存可能具有空间信息 (Fu et al, 2012)。

基于新棘的形成速度和存活比例在动物一生 中保持不变, 可以推测日常形成的新树突棘平均寿 命为 1.5 天, 其中小部分的平均寿命为 $1 \sim 2$ 月, 而 $<0.8 \%$ 的棘在标准饲养环境中可以存在 73 个月 (Yang et al, 2009)。两天训练过程中形成的并能长期 稳定存在的新树突棘占总树突棘数量的比例 $<0.04 \%$, 假设动物寿命为 36 个月, 那么学习引起并 能长期存在的新棘数量为 $2 \times 10^{6}$, 数量足够大, 对 神经环路重新构建以及动物行为具有重要和终生 的影响(Yang et al, 2009)。同时 78\%的旧棘能从一 月龄维持到二月龄, 如果动物生命周期为 36 个月, 一月龄即已存在的棘中 $48 \%$ 能持续到动物生命终 点; 此外，四月龄成年小鼠在标准环境中有 $86 \%$ 的 棘能存在 5 个月(Yang et al, 2009); 因此, 出生后一
个月内形成的大部分树突棘能持续存在一生, 因而 也为基本皮层功能和终生记忆储存提供了结构基 础(Yang et al, 2009)。虽然神经环路存在持续的可塑 性变化, 但是神经环路中存在着两类稳定的树突 棘: 一类是由新经历引起并长久存在的新棘 $(<0.04 \%)$; 另一类来自于出生后早期大量形成并在 发育过程中由不同经历修饰且能长久存在的棘; 由 于这两类树突棘的平均寿命在 70 90 个月左右, 因 而这些树突棘的 $60 \%$ 70\%能在动物的一生中持续 存活, 并直接提供神经环路的终生记忆(Yang et al, 2009)。

在鸣禽的歌唱学习模型中, 发现敏感期或关键 期内的斑胸草雀(Zebra Finch)负责听觉信息与控制 歌唱动作的交叉脑区-前脑 HVC 神经元的树突棘, 在唱歌学习前表现较高的活性, 而歌唱学习可以引 起 HVC 神经元树突棘的快速稳定, 以及数量的积 累和体积的增大; 歌唱学习前树突棘的更新程度越 
高, 动物稍后模仿唱歌的学习能力越强; 通过记录 胞内电活动发现学习过程中的神经活动也显著提 高, 因此树突棘的快速稳定以及突触连接的增强发 生在学习开始前, 可能对于控制学习行为非常重要 (Roberts et al, 2010)。

\section{6 抑制性中间神经元的树突可塑性}

占成熟大脑皮层神经元种类 $20 \%$ 30\%的抑制 性中间神经元, 一般在本区域内进行树突分叉, 并 在局部神经环路中起调控作用, 但是大部分新皮层 中的中间神经元树突轴上没有树突棘(Markram et al, 2004)。关于中间神经元结构可塑性的活体研究 不多, 利用活体双光子成像发现，皮层中间神经元 的树突分支活动性较强(Lee et al, 2006), 嗅球颗粒 细胞的树突分支表现一定的动态特征 (Mizrahi, 2007)。利用 RNA 干扰 $\mathrm{Na}^{+}$通道活性从而降低嗅球 颗粒细胞兴奋性或通过堵鼻子等方法减少嗅觉输 入, 都能引起嗅球中这种抑制性新生中间神经元树 突棘密度的下降(Dahlen et al, 2011; Saghatelyan et al，2005)。单眼剥夺和双眼剥夺能引起中间神经元 动态变化的树突分支的比例增加了 3 倍, 与兴奋性 锥体神经元类似，感觉剥夺期间每周 $10 \%$ 的中间 神经元突触发生更新(Chen \& Nedivi, 2010)。因而推 测成熟大脑皮层中的兴奋性和抑制性突触可能具 有相同程度的结构可塑性(Fu \& Zuo, 2011)。

\section{2 轴突可塑性}

轴突是神经元信息的输出结构。轴突结(axonal boutons) 是突触前结构, 具有不同的形态: 中途结 (en passant boutons)是沿着轴突纤维上的小的膨起 (varicosities); 终端结(terminaux boutons)是分布在 轴突末端的膨起, 类似于球状头部的树突棘 (De Paola et al, 2006)。虽然在小鼠的丘脑皮层中发现轴 突分枝以及形态尺寸在几个月内都能保持稳定，但 是不同类型神经元轴突结的形态和活动性都呈现 不同的特点(De Paola et al, 2006)。在成年猴初级视 皮层中也发现绝大多数轴突分支形式不变, 只有少 量较短分枝上的终端结和少量的中途结每周有更 新, 轴突结形成和消亡的数量占总数的 $7 \%$, 整体 密度无变化(Stettler et al, 2006)。对于感觉损毁的反 应, 皮层神经元轴突也经历着快速的较大范围的改 变, 即使是在成年阶段。恒河猴双眼剥夺一周后, 初级视皮层内朝向损毁投射脑区的轴突生长增加 2 倍; 1 个月后, 由于轴突结的修饰和生长现象同时存
在, 其轴突浓度才开始下降, 但也保持了轴突数量 的净增加; 同时, 轴突结的更新也开始上升 (Yamahachi et al, 2009)。成年小鼠胡须剪掉后体感 皮层内兴奋性和抑制性神经元的轴突呈现大量和 快速的变化, 并伴随轴突结浓度的快速上升; 兴奋 性神经元呈水平投射的轴突净增加, 投射范围从未 剪掉胡须的桶状皮层柱延伸到剪掉胡须的桶状皮 层柱; 剪掉胡须的桶状皮层柱内的抑制性神经元内, 只有靠近胞体附近的轴突有缩回, 其它大多数轴突 可以超过正常范围地远距离投射到周围没有剪掉 胡须的柱状皮层中，并保持稳定; 这也说明兴奋性 和抑制性的平衡是感觉剥夺后拓扑重构的基础 (Marik et al, 2010)。从上述实验中可见成年动物大 脑中轴突分枝非常稳定，同时，轴突也保留着一定 程度的结构可塑性。但是总体而言, 轴突结的活动 性程度低于树突棘(De Paola et al, 2006), 这也解释 了有些新的树突棘不能形成突触，而有些新的树突 棘与已存在的轴突结形成突触(Fu \& Zuo, 2011)。

\section{3 结构可塑性的细胞和分子机制}

突触强度和数量的变化是紧密关联、互相依赖 的, 在活动调控突触形成和丢失的过程中, 突触增 强先于突触数量的增加, 突触减弱先于突触数量的 减少(Leslie \& Nedivi, 2011)。因此, 结构可塑性与功 能可塑性可能具有部分重叠的细胞和分子机制。

据现有的体内和体外实验发现，LTP 过程中， 单个树突棘内发生着肌动蛋白聚合或解聚、细胞膜 重新分布、局部蛋白合成以及谷氨酸受体的胞吞和 胞吐等细胞过程(Kennedy \& Ehlers, 2006), 最终效 应即为在突触后膜中插入 AMPA 型谷氨酸受体、增 强突触连接的强度、以及使被激活的树突棘体积增 大或形成新的树突棘(Derkach et al, 2007)。LTP 信 号起始于 $\mathrm{Ca}^{2+}$ 通过 NMDA 型谷氨酸受体进入突触 后, 使树突棘内 $\mathrm{Ca}^{2+}$ 浓度上升, 从而激活了许多信 号蛋白, 包括蛋白激酶 C (PKC)、 $\mathrm{Ca}^{2+} /$ 钙调素-依赖 的激酶 II (CaMKII)、以及小 $\mathrm{G}$ 蛋白(small GTPase proteins)(如 Ras 和 Rho), 而这些信号分子又引发了 上述细胞活动过程, 进而产生结构和功能的可塑性 (Kennedy et al, 2005)。最近的体外实验证明了上述 信号通路参与了谷氨酸释放诱发树突棘形成的过 程(Kwon \& Sabatini, 2011)。

神经活动不仅能控制神经元的结构和功能的 适应性变化, 同时也能改变基因的表达模式, 因为 
长期记忆、LTP 或 LTD 以及发育过程中神经环路 的修饰都依赖于新的 RNA 和蛋白合成，表明活动 调控的基因也参与了神经可塑性的调控(Leslie \& Nedivi, 2011)。神经活动触发 $\mathrm{Ca}^{2+}$ 内流, 激活一系列 信号传导通路, 并进入核内活化 cAMP 反应元素结 合蛋白(CREB)、血清应答因子和肌细胞增强因子 -2 等一系列转录因子, 进而启动新的基因表达(Greer \& Greenberg, 2008)。据估计, 大脑中受活动调控的 基因约有 500 1 000 个, 其编码产物包括转录因子、 信号转导蛋白、神经营养因子, 以及突触结构和功 能蛋白等可以直接或间接控制神经元形态和功能 的蛋白(Nedivi et al, 1993)。神经活动一般可以引起 细胞核内前后两个阶段的转录反应, 即早基因 (immediate early genes, IEGS)转录反应时期和延迟 早期基因(the delayed early genes)转录反应时期, 但 由于前者一般是转录因子, 促进后者的转录反应, 加之即早基因的表达不具有时间和空间特异性, 大 部分基因功能研究偏重于后者。其中, 参与调节突 触连接增强和减弱的有: 基因信号调节蛋白 2 基因 (regulator of gene signaling 2, rgs2)、活动调控的细胞 骨架伴生蛋白基因 (activity-regulated cytoskeletonassociated protein, arc/arg3.1)、可塑性相关基因 2(candidate plasticity gene 2, cpg2)、Homer 同源物 1a 基因(Homer homolog 1a, homer1a)和血清诱导激 酶基因(serum-induced kinase, snk); 调节突触数量 增加和减少的基因有: 可塑性相关基因 15 (candidate plasticity gene 15, cpg15)、组织型纤溶酶 原激活剂基因 (tissue-type plasminogen activator, $\mathrm{tPA}$ )、活动调控的类钻黏着蛋白 (activity regulated cadherin-like protein, arcadlin)、神经元 PAS 域蛋白 4 基因(neuronal PAS domain protein 4, npas4)和神经 活动调节穿透素基因 (neuronal activity-regulated pentraxin, narp); 也有直接调节突触形态的基因, 如 脑源性神经营养因子 (brain-derived neurotrophic factor, bdnf)等(Leslie \& Nedivi, 2011)。

除特定基因的活动依赖性调控表达外, 还有活 动依赖性的转录后调控, 包括: mRNA 从胞体运输 到树突和轴突的调控、突触活动在树突引起局部翻 译活动的调控、活动依赖型胞外蛋白分泌的调控、 活动调控的 mRNA 降解等(Leslie \& Nedivi, 2011)。 总体而言, 神经活动激活了从突触到胞核的信号通 路, 并启动一系列新的基因表达程序, 这些基因产
物通过一系列细胞过程来实现神经功能和结构的 改变, 包括突触增强或减弱以及突触形成或消亡, 调整原有神经环路和形成新的神经连接, 这就是活 动依赖的神经可塑性发生的整个过程(Leslie \& Nedivi, 2011)。

\section{4 研究展望}

虽然对神经环路的结构可塑性及其分子细胞 机制的研究已经深入到突触水平，但是很多问题仍 未完全解决。哪些信号能触发树突棘的增大和萎缩, 哪些信号可以调控树突棘的更新, 并怎样相互协 调? 哪些因素可以使树突棘变得稳定? 稳定的磨 菇状树突棘还对神经活动敏感吗? 不同大脑皮层 以及同一皮层不同层内树突棘稳定性的差异是来 自于神经元本身的差异, 还是皮层局部活动模式的 差异? 树突棘形态或更新异常的机制是什么? 树 突棘可塑性的改变如何导致临床症状和疾病进 展? 结构可塑性与突触的功能变化相关性多大? 突触结构的变化对神经环路的改变起多大作用? 神经结构的动态变化是行为改变的原因还是结 果? 程序性记忆的巩固可能与树突棘的稳定相关, 那么陈述性记忆巩固的结构基础是否类似? 动态 变化的神经环路如何维持永久的记忆? 活动依赖 型相关基因与功能? 关键期可塑性的作用到底是 什么(Alvarez \& Sabatini, 2007; Fu \& Zuo, 2011; Kennedy \& Ehlers, 2006)?

现有的双光子显微成像技术只能观察到树突 棘的更新, 不能准确观察到树突棘形态变化的程度 以及该树突棘是否具有功能等, 因此需要结合其它 技术, 包括神经活动标志物(如 $\mathrm{Ca}^{2+}$ 敏感荧光蛋白)、 可以进行特异性活动调控的光遗传学标记及电镜 等。此外, 树突棘和轴突结作为突触前后的相关 结构, 其可塑性是否相同, 是否在相同活动中变 化一致均不清楚, 因此需要同时监测两者的结构 变化。

总之, 一个具有功能的成熟的神经系统是从最 初的一群不精确的突触连接通过选择性地建立及 消除一些突触的过程来完成的，这些过程是基于基 因与经历的共同调节(Bhatt et al, 2009)。通过研究神 经环路的结构可塑性, 为我们更深入的理解神经的 发育、学习和记忆以及神经和精神疾病机制提供了 重要信息。 


\section{参考文献:}

Alvarez VA, Sabatini BL. 2007. Anatomical and physiological plasticity of dendritic spines [J]. Annu Rev Neurosci, 30: 79-97.

Amaral MD, Pozzo-Miller L. 2009. The dynamics of excitatory synapse formation on dendritic spines [J]. Cellscience, 5(4): 19-25.

Bear MF, Huber KM, Warren ST. 2004. The mGluR theory of fragile X mental retardation [J]. Trends Neurosci, 27(7): 370-377.

Bhatt DH, Zhang SX, Gan WB. 2009. Dendritic spine dynamics [J]. Annu Rev Physiol, 71: 261-282.

Bourne J, Harris KM. 2007. Do thin spines learn to be mushroom spines that remember [J]? Curr Opin Neurobiol, 17(3): 381-386.

Chen JL, Nedivi E. 2010. Neuronal structural remodeling: is it all about access [J]? Curr Opin Neurobiol, 20(5): 557-562.

Chklovskii DB, Mel BW, Svoboda K. 2004. Cortical rewiring and information storage [J]. Nature, 431(7010): 782-788.

Dahlen JE, Jimenez DA, Gerkin RC, Urban NN. 2011. Morphological analysis of activity-reduced adult-born neurons in the mouse olfactory bulb [J]. Front Neurosci, 5: 66.

De Paola V, Holtmaat A, Knott G, Song S, Wilbrecht L, Caroni P, Svoboda K. 2006. Cell type-specific structural plasticity of axonal branches and boutons in the adult neocortex [J]. Neuron, 49(6): 861-875.

Derkach VA, Oh MC, Guire ES, Soderling TR. 2007. Regulatory mechanisms of AMPA receptors in synaptic plasticity [J]. Nat Rev Neurosci, 8(2): 101-113.

Engert F, Bonhoeffer T. 1999. Dendritic spine changes associated with hippocampal long-term synaptic plasticity [J]. Nature, 399(6731): 66-70.

Fox K, Wong ROL. 2005. A comparison of experience-dependent plasticity in the visual and somatosensory systems [J]. Neuron, 48(3): 465-477.

$\mathrm{Fu} \mathrm{M,} \mathrm{Yu} \mathrm{XZ,} \mathrm{Lu} \mathrm{J,} \mathrm{Zuo} \mathrm{Y.} \mathrm{2012.} \mathrm{Repetitive} \mathrm{motor} \mathrm{learning} \mathrm{induces}$ coordinated formation of clustered dendritic spines in vivo [J]. Nature, 483(7387): 92-95

Fu M, Zuo Y. 2011. Experience-dependent structural plasticity in the cortex [J]. Trends Neurosci, 34(4): 177-187.

Greer PL, Greenberg ME. 2008. From synapse to nucleus: calcium-dependent gene transcription in the control of synapse development and function [J]. Neuron, 59(6): 846-860.

Grutzendler J, Kasthuri N, Gan WB. 2002. Long-term dendritic spine stability in the adult cortex [J]. Nature, 420(6917): 812-816.

Hao JD, Rapp PR, Leffler AE, Leffler SR, Janssen WGM, Lou W, McKay H, Roberts JA, Wearne SL, Hof PR, Morrison JH. 2006. Estrogen alters spine number and morphology in prefrontal cortex of aged female rhesus monkeys [J]. J Neurosci, 26(9): 2571-2578.

Ho VM, Lee JA, Martin KC. 2011. The cell biology of synaptic plasticity [J] Science, 334(6056): 623-628.

Holtmaat A, Svoboda K. 2009. Experience-dependent structural synaptic plasticity in the mammalian brain [J]. Nat Rev Neurosci, 10(9): 647-658

Holtmaat A, Wilbrecht L, Knott GW, Welker E, Svoboda K. 2006. Experience-dependent and cell-type-specific spine growth in the neocortex [J]. Nature, 441(7096): 979-983.

Holtmaat AJGD, Trachtenberg JT, Wilbrecht L, Shepherd GM, Zhang XQ, Knott GW, Svoboda K. 2005. Transient and persistent dendritic spines in the neocortex in vivo [J]. Neuron, 45(2): 279-291.

Kasai H, Fukuda M, Watanabe S, Hayashi-Takagi A, Noguchi J. 2010. Structural dynamics of dendritic spines in memory and cognition [J]. Trends Neurosci, 33(3): 121-129.

Kaufmann WE, Moser HW. 2000. Dendritic anomalies in disorders associated with mental retardation [J]. Cereb Cortex, 10(10): 981-991.
Kennedy MB, Beale HC, Carlisle HJ, Washburn LR. 2005. Integration of biochemical signalling in spines [J]. Nat Rev Neurosci, 6(6): 423-434.

Kennedy MJ, Ehlers MD. 2006. Organelles and trafficking machinery for postsynaptic plasticity [J]. Annu Rev Neurosci, 29: 325-362.

Kwon HB, Sabatini BL. 2011. Glutamate induces de novo growth of functional spines in developing cortex [J]. Nature, 474(7349): 100-104.

Lee WC, Huang H, Feng GP, Sanes JR, Brown EN, So PT, Nedivi E. 2006. Dynamic remodeling of dendritic arbors in GABAergic interneurons of adult visual cortex [J]. PLoS Biol, 4(2): e29.

Lendvai B, Stern EA, Chen B, Svoboda K. 2000. Experience-dependent plasticity of dendritic spines in the developing rat barrel cortex in vivo [J]. Nature, 404(6780): 876-881.

Leslie JH, Nedivi E. 2011. Activity-regulated genes as mediators of neural circuit plasticity [J]. Prog Neurobiol, 94(3): 223-237.

Majewska A, Sur M. 2003. Motility of dendritic spines in visual cortex in vivo: changes during the critical period and effects of visual deprivation [J]. Proc Natl Acad Sci USA, 100(26): 16024-16029.

Marik SA, Yamahachi H, McManus JNJ, Szabo G, Gilbert CD. 2010. Axonal dynamics of excitatory and inhibitory neurons in somatosensory cortex [J]. PLoS Biol, 8(6): e1000395.

Markram H, Toledo-Rodriguez M, Wang Y, Gupta A, Silberberg G, Wu CZ. 2004. Interneurons of the neocortical inhibitory system [J]. Nat Rev Neurosci, 5(10): 793-807.

Mataga N, Mizuguchi Y, Hensch TK. 2004. Experience-dependent pruning of dendritic spines in visual cortex by tissue plasminogen activator $[\mathrm{J}]$. Neuron, 44(6): 1031-1041.

Matsuzaki M, Honkura N, Ellis-Davies GCR, Kasai H. 2004. Structural basis of long-term potentiation in single dendritic spines [J]. Nature, 429(6993): 761-766.

Mizrahi A. 2007. Dendritic development and plasticity of adult-born neurons in the mouse olfactory bulb [J]. Nat Neurosci, 10(4): 444-452.

Mizrahi A, Crowley JC, Shtoyerman E, Katz LC. 2004. High-resolution in vivo imaging of hippocampal dendrites and spines [J]. J Neurosci, 24(13): 3147-3151.

Mizrahi A, Katz LC. 2003. Dendritic stability in the adult olfactory bulb [J]. Nat Neurosci, 6(11): 1201-1207.

Mozzachiodi R, Byrne JH. 2010. More than synaptic plasticity: role of nonsynaptic plasticity in learning and memory [J]. Trends Neurosci, 33(1): 17-26

Nedivi E, Hevroni D, Naot D, Israeli D, Citri Y. 1993. Numerous candidate plasticity-related genes revealed by differential cDNA cloning [J]. Nature, 363(6431): 718-722.

Oray S, Majewska A, Sur M. 2004. Dendritic spine dynamics are regulated by monocular deprivation and extracellular matrix degradation $[\mathrm{J}]$. Neuron, 44(6): 1021-1030.

Pan F, Aldridge GM, Greenough WT, Gan WB. 2010. Dendritic spine instability and insensitivity to modulation by sensory experience in a mouse model of fragile X syndrome [J]. Proc Natl Acad Sci USA, 107(41): 17768-17773.

Park M, Salgado JM, Ostroff L, Helton TD, Robinson CG, Harris KM, Ehlers MD. 2006. Plasticity-induced growth of dendritic spines by exocytic trafficking from recycling endosomes [J]. Neuron, 52(5): 817-830.

Patterson M, Yasuda R. 2011. Signalling pathways underlying structural plasticity of dendritic spines [J]. Br J Pharmacol, 163(8): 1626-1638.

Roberts TF, Tschida KA, Klein ME, Mooney R. 2010. Rapid spine stabilization and synaptic enhancement at the onset of behavioural learning [J]. Nature, 463(7283): 948-952. 
Saghatelyan A, Roux P, Migliore M, Rochefort C, Desmaisons D, Charneau P, Shepherd GM, Lledo PM. 2005. Activity-dependent adjustments of the inhibitory network in the olfactory bulb following early postnatal deprivation [J]. Neuron, 46(1): 103-116.

Stettler DD, Yamahachi H, Li W, Denk W, Gilbert CD. 2006. Axons and synaptic boutons are highly dynamic in adult visual cortex [J]. Neuron, 49(6): 877-887.

Toni N, Buchs PA, Nikonenko I, Bron CR, Muller D. 1999. LTP promotes formation of multiple spine synapses between a single axon terminal and a dendrite [J]. Nature, 402(6760): 421-425.

Trachtenberg JT, Chen BE, Knott GW, Feng GP, Sanes JR, Welker E, Svoboda K. 2002. Long-term in vivo imaging of experience-dependent synaptic plasticity in adult cortex [J]. Nature, 420(6917): 788-794.

Tsai J, Grutzendler J, Duff K, Gan WB. 2004. Fibrillar amyloid deposition leads to local synaptic abnormalities and breakage of neuronal branches [J]. Nat Neurosci, 7(11): 1181-1183.

Xu TH, Yu XZ, Perlik AJ, Tobin WF, Zweig JA, Tennant K, Jones T, Zuo Y. 2009. Rapid formation and selective stabilization of synapses for enduring motor memories [J]. Nature, 462(7275): 915-919.
Yamahachi H, Marik SA, McManus JNJ, Denk W, Gilbert CD. 2009. Rapid axonal sprouting and pruning accompany functional reorganization in primary visual cortex [J]. Neuron, 64(5): 719-729.

Yang G, Pan F, Gan WB. 2009. Stably maintained dendritic spines are associated with lifelong memories [J]. Nature, 462(7275): 920-924.

Yuste R, Bonhoeffer T. 2004. Genesis of dendritic spines: insights from ultrastructural and imaging studies [J]. Nat Rev Neurosci, 5(1): 24-34.

Zhou Q, Homma KJ, Poo MM. 2004. Shrinkage of dendritic spines associated with long-term depression of hippocampal synapses [J]. Neuron, 44(5): 749-757.

Ziv NE, Ahissar E. 2009. Neuroscience: New tricks and old spines [J]. Nature, 462(7275): 859-861

Zuo Y, Lin A, Chang P, Gan WB. 2005a. Development of long-term dendritic spine stability in diverse regions of cerebral cortex $[\mathrm{J}]$. Neuron, 46(2): 181-189.

Zuo Y, Yang G, Kwon E, Gan WB. 2005b. Long-term sensory deprivation prevents dendritic spine loss in primary somatosensory cortex [J]. Nature, 436(7048): 261-265.

\section{中国科学院武汉物理与数学研究所神经影像实验室简介}

中国科学院武汉物理与数学研究所神经影像实验室建立于 2007 年 4 月, 由国家杰出青年基金获得者 徐富强研究员领导。综合不同层次和尺度的神经生物学的方法和技术是该实验室的显著特点。现已建立分 子生物学、生物化学、神经环路示踪、微透析、电生理、光学成像、磁共振成像、动物行为等方法和技术, 拟建立微观的分子/细胞水平研究与宏观的系统/行为研究之间的桥梁，以解决气味信息的编码机制以及重 大神经疾病的神经环路基础等基本问题。目前, 该实验室有 2 名助理研究员, 2 名博士后, 2 名实验师和 14 名研究生。其主要研究课题和方向为: 嗅觉编码机制研究; 生理状态调控气味感知的研究; 㖵觉与老年 痴呆病的相关研究; 神经环路的病毒标记技术研究; 嗅觉与饮食关系的研究等。本实验室已与耶鲁大学、 普林斯顿大学、纽约大学、科罗拉多大学, 北京生命科学研究所, 中科院生物物理所、中科院昆明动物研 究所、第二军医大学、华中科技大学、武汉大学、华中农业大学等国内外研究机构均已建立紧密的合作关 系。

\section{近期发表的主要文章有:}

1. R. Gong, C. Ding, J. Hu, Y. Lu, F. Liu, E. Mann, F. Xu, MB. Cohen and M. Luo, Role for the Membrane Receptor Guanylyl Cyclase-C in Attention Deficiency and Hyperactive Behavior. Science, 333(6049):1642-6 (2011).

2. A. Li, L. Gong, F. Xu ${ }^{*}$. Brain-state-independent neural representation of peripheral stimulation in olfactory bulb. PNAS, 108:5087-92 (2011).

网址: http://www.wipm.ac.cn/jgsz/yjdw/cigongzhenyingyong/shenjingyingxiang/yanjiuneirong/ 地址：湖北省武汉市武昌区八一路小洪山西 30 号

邮编: 430071 